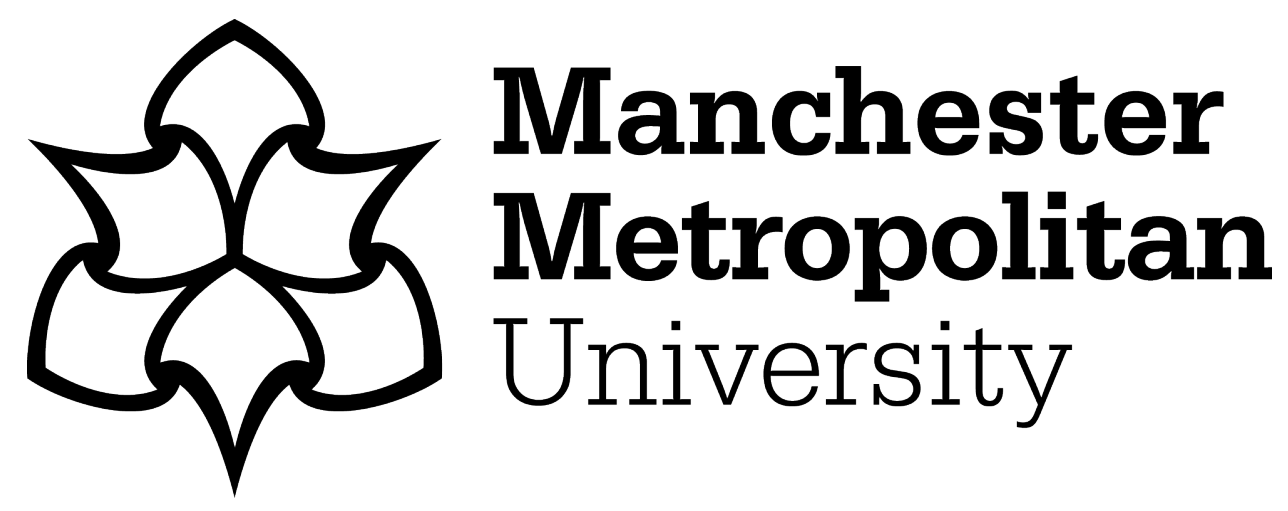

Tobias, JH, Sayers, A, Deere, KC, Heazell, AEP, Lawlor, DA and Ireland, A ORCID logoORCID: https://orcid.org/0000-0003-1094-9183 (2019) Breech presentation is associated with lower adolescent tibial bone strength. Osteoporosis International, 30. pp. 1423-1432. ISSN 0937-941X

Downloaded from: https://e-space.mmu.ac.uk/622883/

Version: Accepted Version

Publisher: Springer (part of Springer Nature)

DOI: https://doi.org/10.1007/s00198-019-04945-4

Please cite the published version 


\section{Breech presentation is associated with lower adolescent tibial bone strength}

Jon H Tobias ${ }^{1}$, Adrian Sayers ${ }^{1}$, Kevin C Deere ${ }^{1}$, Alexander EP Heazell ${ }^{2}$, Debbie A Lawlor ${ }^{3,4,5}$ \& Alex Ireland ${ }^{6}$

1. Musculoskeletal Research Unit, Translational Health Sciences, Bristol Medical School, University of Bristol

2. Division of Developmental Biology and Medicine, Faculty of Biology, Medicine and Health, University of Manchester, UK

3. MRC Integrative Epidemiology Unit at the University of Bristol, Bristol, UK.

4. Population Health Science, Bristol Medical School, Bristol University, UK

5. Bristol NIHR Biomedical Research Centre

6. School of Healthcare Science, Manchester Metropolitan University, Manchester, UK.

\section{Corresponding Author}

Alex Ireland, Manchester Metropolitan University, John Dalton Building, Chester Street, Manchester, M1 5GD. a.ireland@mmu.ac.uk. +44 (0)161 2471987.

\section{Author Disclosures}

Debbie A Lawlor declares that she has received research support from several national and international government and charitable funders, and Roche Diagnostics and Medtronic for research unrelated to that presented in this paper. Jon H Tobias, Adrian Sayers, Kevin C Deere, Alexander EP Heazell, and Alex Ireland declare that they have no conflict of interest.

\section{Mini Abstract}

We compared bone outcomes in adolescents with breech and cephalic presentation. Tibia bone mineral content, density, periosteal circumference and cross-sectional moment of inertia were lower in breech presentation, and females with breech presentation had lower hip CSA. These findings suggest that prenatal loading may exert long-lasting influences on skeletal development. 


\begin{abstract}
Purpose: Breech position during pregnancy is associated with reduced range of fetal movement, and with lower limb joint stresses. Breech presentation at birth is associated with lower neonatal bone mineral content (BMC) and area, but it is unknown whether these associations persist into later life.

Methods: We examined associations between presentation at onset of labour, and tibia and hip bone outcomes at age 17 years in 1971 participants (1062 females) from a UK prospective birth cohort that recruited $>15,000$ pregnant women in 1991-1992. Cortical BMC, cross-sectional area (CSA) and bone mineral density (BMD), periosteal circumference, and cross-sectional moment of inertia (CSMI) were measured by peripheral quantitative computed tomography (pQCT) at $50 \%$ tibia length. Total hip $B M C$, bone area, BMD and CSMI were measured by dual-energy $X$-ray absorptiometry (DXA).

Results: In models adjusted for sex, age, maternal education, smoking, parity, and age, singleton/multiple births, breech presentation ( $n=102$ ) was associated with lower tibial cortical BMC $(-0.14 S D, 95 \% \mathrm{Cl}-0.29$ to 0.00$)$, CSA (-0.12SD, -0.26 to 0.02$)$, BMD (-0.16SD, -0.31 to -0.01$)$, periosteal circumference (-0.14SD, -0.27 to -0.01 ) and CSMI (-0.11SD, -0.24 to 0.01$)$. In females only, breech presentation was associated with lower hip CSA (-0.24SD, -0.43 to 0.00$)$ but not with other hip outcomes. Additional adjustment for potential mediators (delivery method, birthweight, gestational age, childhood motor competence and adolescent height and body composition) did not substantially affect associations with either tibia or hip outcomes.

Conclusions: These findings suggest that prenatal skeletal loading may exert long-lasting influences on skeletal size and strength but require replication.
\end{abstract}

\title{
Keywords
}

ALSPAC, fetal movement, pregnancy, osteoporosis, bone mass 


\section{Introduction}

Identification of factors influencing bone mass and strength in childhood is important, as peak bone mass is a major determinant of osteoporosis risk in later life [1]. Skeletal growth is most rapid prenatally, with the skeleton reaching around $45 \mathrm{~cm}$ in length by 9 months gestation, a rate unmatched even during the pubertal growth spurt [2]. Bone mass tracks across childhood [3], therefore it is unsurprising that key factors influencing perinatal bone mass accrual such as birthweight [4] also associate with bone mass and strength in adolescence [5]. Gestation length remains positively associated with bone mass into older age [6], and is inversely associated with fracture risk [7]. Therefore, identifying prenatal factors influencing bone development is important for the development and maintenance of bone health.

One of the strongest predictors of childhood and adult bone health is skeletal loading via physical activity [8]. Evidence from in silico [9] and animal [10] models suggests a key role for skeletal loading in fetal bone and joint development. In humans, neuromuscular diseases causing fetal immobility result in slender, thin-walled, fracture-prone bones [11], but evidence for effects of prenatal skeletal loading in healthy individuals is limited. This is likely due to difficulties in measuring components of movement relevant to bone health in utero, and thereby identifying reduced fetal skeletal loading in otherwise healthy fetuses.

Babies occupying a breech position during pregnancy have restricted movement of the lower limbs in the third trimester [12]. Whilst the number of movements does not appear to differ [13], this reduced range of movement is likely to contribute to reduced skeletal loading of the lower limbs in breech position [14] during late pregnancy, a period when the stresses placed upon the growing skeleton are usually greatest [15]. Therefore breech position during pregnancy could be considered as a natural (non-pathological) model of reduced fetal movement and skeletal loading. The skeletal consequences of this reduced loading are evident at birth, with a ten-fold increase in the incidence of hip dyplasia [16], $10^{\circ}$ greater femoral anteversion [17] and greater patellofemoral joint dyplasia [18] in babies born breech than in cephalic or conventional presentation. Bone mass accrual is also affected, with lower neonatal bone mass and area in breech babies [19]. Postnatally, reduced active and locomotory movements [20], a restricted range of joint motion and altered joint position [11, 20-23] and shorter periods of sustained movements [24] have been observed in breech presentation. Effects on motor ability in later life are unclear [21,25], although there may be differences in gait [25]. However, whilst there is some evidence of lower hip bone mass in breech babies in early childhood [19], it is unknown whether these deficits are still evident in later life closer to the time of peak bone mass. Given that around $50 \%$ of fetuses are in a breech position at 25 weeks' gestation [26] with $4-5 \%$ remaining in breech presentation at birth [27], such deficits could have longer term implications for a large number of otherwise healthy children.

In the current study we examined associations between breech presentation at birth and lower limb bone outcomes in late adolescence in a large, population-based birth cohort. We hypothesised that lower bone mass and area would be evident in adolescents with a history of breech rather than cephalic presentation. 


\section{Methods}

The Avon Longitudinal Study of Parents and Children (ALSPAC) is a geographically-based birth cohort study located in and around the Bristol area in the UK that investigates genetic, environmental, and social influences on health and development of children and young people [28, 29]. All pregnant women resident in the former Avon Health Authority in South West England having a delivery date between April 1, 1991, and December 31, 1992 were invited to take part resulting in a cohort of 15,247 pregnancies and 14,701 children alive at 12 months [28]. Ethical approval for the study was obtained from the ALSPAC Ethics and Law Committee and the Local Research Ethics Committees. The present study is based on data collected from obstetric medical notes, antenatal and postnatal questionnaires, and the adolescence research clinic undertaken when participants were at a mean age of 17 years. Written informed consent was provided by parents, and young people provided written assent. Please note that the study website (http://www.bristol.ac.uk/alspac/researchers/our-data/) contains details of all the data that is available through a fully searchable data dictionary and variable search tool.

\section{Exposure measure: Fetal position at onset of labour}

Fetal position at onset of labour (breech or cephalic) was obtained from obstetric records. Participants were included irrespective of mode of delivery (though this was adjusted for), as the exposure here is related to position at the end of pregnancy. 103 individuals with a fetal presentation other than breech or cephalic (e.g. transverse lie) were excluded from analyses).

Outcome measures: peripheral quantitative computed tomography ( $P Q C T)$ and dual-energy X-ray absorptiometry (DXA)

All offspring who attended the ALSPAC research clinic at 17 years old were offered a pQCT scan at the 50\% tibia length site using an XCT 2000 scanner (Stratec, Pforzheim, Germany). Measurements were analyzed and results exported using the Automated Analysis Tools in Version 6.00B of the software supplied with the machine. A threshold of $650 \mathrm{mg} / \mathrm{mm}^{3}$ was used to separate cortical bone; this threshold has been shown to accurately assess bone geometry [30]. From these analyses, details of cortical bone mineral content (BMC), cortical bone mineral density (BMD) and cortical cross-sectional area (CSA) were recorded together with periosteal circumference and cross-sectional moment of inertia (CSMI). In addition, to obtain a measure of muscle size, images were filtered using the in-built F03F05F05 filter, before a threshold of $30 \mathrm{mg} / \mathrm{mm}^{3}$ was used to remove fat from the image, and calculated total bone area was subtracted to derive muscle CSA.

Participants were also offered total body and hip dual-energy X-ray absorptiometry (DXA) scans using a GE Lunar Prodigy (Madison, Wisconsin) in standard scanning mode. From total body scans, lean mass and fat mass in kg were recorded, whilst hip BMD, BMC and bone area (BA) were measured from hip scans. The manufacturer's automated advanced hip analysis (AHA) software was used to measure CSMI at the site of minimal femoral neck width. Error codes were generated for positioning, artefact and movement errors for each variable.

Short-term error was assessed as coefficient of variation (CV) between repeated pQCT scans in 126 individuals, and between repeated DXA scans in 153 individuals. CV for cortical BMC was 2.6\%, cortical BMD 1.1\%, periosteal circumference 1.5\% and CSMI 5.8\%, whilst total hip BMD CV was $1.2 \%$ and hip CSMI 7.5\%. 


\section{Covariables:}

In this analysis, maternal socioeconomic position (assessed by maternal educational attainment), smoking during pregnancy, age, parity and whether the pregnancy was singleton/multiple were considered as potential confounders based on their plausible effects on both fetal position and bone outcomes. Data on these were obtained from questionnaires completed by the mother prior to offspring birth. Delivery method, length of gestation and birthweight were considered potential mediators as these may be influenced by fetal position and subsequently influence bone outcomes. Data on these were obtained from obstetric records. We also adjusted for exact age at the 17 year follow up, as well as sex, to improve statistical efficiency. We also considered height, body composition and motor abilities as potential mediators due to previous reports of impaired growth and motor development in children born with breech presentation. Height was measured using a Harpenden stadiometer (Holtain Ltd., Crymych, UK), and weight was measured to the nearest $50 \mathrm{~g}$ using weighing scales (Tanita UK Ltd, Uxbridge, UK). An estimate of the child's motor abilities was obtained at around 18 months of age using a scale developed by ALSPAC including elements derived from the Denver Developmental Screening Test [31]. Mothers were asked to complete a series of questions as to whether their child regularly, occasionally or had never completed movements such as walking, climbing and jumping. These answers were used to calculate a continuous Gross Motor Score (GMS).

\section{Statistical Analyses:}

Analysis was restricted to those participants with complete data on birth position, bone outcomes and all covariables included in any model ( $N=1971$ ( $38 \%$ of those eligible; Figure 1$)$ ). Differences in basic characteristics and bone outcomes between cephalic and breech presentations were examined by Fisher's exact test, $\chi 2$ tests and t-tests for binary, categorical and continuous variables respectively. Associations between presentation type and bone outcomes were then assessed with multiple linear regression models using the $\mathrm{R}$ statistical environment (version 3.1.2, www.r-project.org). Minimal model 1 was adjusted for height, due to the strong association between size and bone strength. Model 2 was also adjusted for sex, age at outcome and early life confounders i.e. maternal social class, maternal smoking, parity, maternal age and singleton/multiple births. Model 3 was additionally adjusted for possible mediators, namely total body fat mass and lean mass (or muscle CSA in the case of pQCT variables), gestational age, birthweight, delivery method and GMS. Sex interactions were also examined in Models 2 and 3 due to previous reports of sex-dependent associations between early life loading and adolescent bone outcomes. Residual plots were examined to ensure homoscedasticity of residuals; for tibia CSMI only, data were log-transformed to improve model fit. 


\section{Results}

There were 5166 individuals with complete neonatal data including presentation type (Figure 1); of these, 1971 (38\% of eligible) had valid DXA and pQCT scans and complete data on all covariables at age 17 and were included in our analyses. Compared with those included in analyses, those who were excluded were more likely to be male, have a mother who smoked during pregnancy, were less likely to be first-born, had a lower level of maternal education and lower maternal age (Supplementary Table 1). There was no difference in presentation type, singleton/multiple births, birthweight or gestation age between included and excluded participants.

Participant characteristics of those with complete data are shown in Table 1. Breech presentation was associated with greater likelihood of a caesarean birth, lower birthweight, shorter gestation length and greater maternal age. All other characteristics were similar between groups.

Bone outcomes and body composition according to presentation are shown in Table 2; in these unadjusted analyses, tibial cortical BMC, cortical CSA, periosteal circumference and cortical CSMI appeared to be lower in breech than cephalic presentation (all $P>0.05$ ), whereas similar values were seen for tibial cortical BMD, DXA bone outcomes, total body lean and fat mass, and muscle CSA. In the minimally-adjusted model 1 (Figure 2), breech presentation was associated with lower tibial cortical BMC, cortical CSA, periosteal circumference and cortical CSMI. After further adjustments for possible confounders and mediators in Models 2 and 3 , breech presentation was now also associated with $0.18 \mathrm{SD}(0.5 \%)$ lower tibial cortical BMD. Further analysis of individual covariates suggested that this was primarily attributable to adjustment for sex in Model 2 . Therefore sex may be acting as a masking confounder, due to the higher proportion of females with breech presentation and greater cortical BMD in females than males. In contrast, associations between presentation type and tibial cortical BMC (0.18SD or $3.0 \%$ lower in breech than cephalic presentation), cortical CSA (0.15SD or $2.6 \%$ ), periosteal circumference (0.27SD or $1.2 \%$ ) and cortical CSMI (0.25SD or $4.4 \%$ ) remained unchanged following additional adjustments in Model 3 . There was no strong statistical evidence of association between presentation type and DXA bone outcomes in any model (all $P>0.05$, Table 3 ). Given the relatively small number of participants with breech presentation, we were keen to ensure that observed group differences were not attributable to a small number of individuals skewing the group results. Therefore, we examined the distribution of data in the two groups (Supplementary Figure 1) which suggested that outlying results were not likely to be responsible for the associations we observed.

Sex-stratified analyses showed broadly similar results in both sexes, with little evidence of a sex interaction overall (Table 3 and Supplementary Table 2). That said, inverse associations between delivery method and PQCT parameters tended to be stronger in females compared to males. Moreover, the association between breech delivery and hip CSA showed evidence of a sex interaction, with an inverse association in females which was strongest in model 2 (Table 3). 


\section{Discussion}

We investigated associations between presentation immediately prior to birth and bone outcomes in late adolescence. In our confounder-adjusted model, we found that breech presentation was associated with lower tibial cortical BMC, cortical CSA, cortical BMD, periosteal circumference and cortical CSMI (a measure of torsional stiffness). There was some suggestion that these associations were stronger in females, which was particularly evident for hip CSA as measured by DXA, which was lower in females with breech presentation, whereas an equivalent association was not seen in males. However, these sex differences require further replication in other large independent datasets.

\section{Comparison with previous findings}

To our knowledge, this is the first study to examine bone health in late adolescence in individuals born with a breech presentation. A previous study has shown that breech presentation is associated with lower whole-body bone mass and area measured by DXA at birth, and lower hip bone mass and area at age four years [19]. In addition, tibia bone ultrasound velocity in neonates (an indirect indicator of BMD) is lower in those with breech presentation [32]. Breech presentation is associated with lower birthweight and crown-heel length, but it was not found to be associated with height and body composition at age four years [19]. Similarly, in the current study we found no notable differences in adolescent body size (height, body weight, total body lean or fat mass) between those with breech and cephalic presentation at birth.

\section{Possible explanation of findings}

Bone mass and area deficits evident in the tibia of individuals born with a breech presentation could be attributable to a reduced range of lower limb movements and lower skeletal loading $[11,14]$ during a key period for prenatal bone growth [15]. While it is difficult to gain direct evidence to support this conclusion, we found little evidence to support alternative explanations. For example, there was little evidence to suggest a role of altered body composition (which was similar in breech and cephalic presentation), in contrast to findings from a previous study of early postnatal loading and bone health in individuals from the same cohort [33]. Whilst differences in birthweight and gestational age were previously suggested to contribute to those in neonatal bone mass and area following breech presentation [19], this was not the case in the current study.

An alternative explanation could be that observed bone strength deficits reflect pre-existing impairment in neuromuscular development which predisposes the fetus to breech presentation. In addition to limiting the ability to attain a cephalic position [34], reduced prenatal motor development may contribute with altered limb position to lower skeletal loading [12]. Consistent with this possibility, the frequency of breech presentation is higher in children with developmental conditions affecting neuromuscular function such as cerebral palsy [35] and meningocele [34]. However, such conditions are associated with impaired motor development and reduced lean mass, which did not differ substantially between breech and cephalic presentation, and adjustment for these covariates did not attenuate associations between breech presentation and bone outcomes. Breech presentation could also reflect differences in maternal mechanical factors such as the shape, size and 
health of the pelvis and uterus which may also restrict fetal growth and development. These were not measured in the current study, although previous work suggests that they do not substantially contribute to risk of breech presentation [36].

A number of tibia bone outcomes differed between breech and cephalic presentation whereas area was the only hip outcome to differ between groups. Mathematical models of the developing fetal skeleton suggest that lower limb bone stresses caused by fetal kicks are greatest in the tibia and femoral shafts [37]. In addition, the tibia shaft is predominately made up of cortical bone whereas the proximal femur also contains trabecular bone. Therefore, these localised associations may reflect mechanical loading patterns and tissue-specific response, rather than differences in inherent sensitivity of the methods used to assess bone at different sites. Future studies could examine associations between presentation type and bone outcomes in the femoral shaft or tibia epiphyses.

Effect sizes for associations between breech presentation and tibia bone outcomes were larger in females than males. In addition, there was evidence of a sex interaction for hip area with lower values in breech presentation for females only. These findings are in contrast with a previous study where associations between breech presentation and total body and hip DXA bone outcomes at birth and four years of age were similar in males and females [19]. It is important to note that no tibia pQCT measurements were reported in the previous study and caution must be taken when interpreting regional bone outcomes in young children due to potential for greater measurement error. There is some evidence that sex hormone levels are associated with fetal hip development [38], and may contribute to greater risk of developmental hip disorders in females than males [16]. This may be related to the influence of sex hormones such as estrogen on the sensitivity of the skeleton to mechanical stimuli [39], which could explain the interaction between sex and restricted movements in breech on bone. However, it is unknown whether these associations also contribute to observed sex differences in neonatal bone cross-sectional geometry and mass [40]. It is important to note that a large number of statistical comparisons have been undertaken in this paper, interactions (in particular sex interactions) often fail to subsequently replicate in independent samples and we have no evidence of such interactions in previously published studies. Therefore, the sex difference noted here should be treated with caution until replicated in large independent studies.

\section{Significance and implications}

In fully-adjusted models, the effect size of these associations was greater than for a number of established factors that have been shown to relate to bone mass, including fat mass and maternal smoking (Supplementary Figure 2). The PQCT parameters associated with breech presentation largely reflect lower bone mass due to reduction in skeletal size, which is inversely related to fracture risk. It would seem reasonable that, though just measuring at the tibia, these size effects are generalised hence fracture risk at other lower limb sites may be affected. The association with hip CSA in females would be consistent with this, although the more common clinical measure of hip BMD was not associated with presentation type in either sex. These results could add weight to the theory of intrauterine programming i.e. that adverse in utero environment has long-term consequences for health, for which a limited number of examples exist for bone [41]. 
Breech presentation represents a potentially modifiable factor, as effective interventions to reverse breech presentation are available [42]. However, whilst they substantially reduce the incidence of hip dysplasias in breech presentation [43] it is unknown whether they affect bone mass and size at birth. Unfortunately, only fourteen attempts at manual cephalic version were recorded in this cohort with no details on success rates or reversion to breech presentation, therefore we were unable to explore the impact of attempted cephalic version on the observed associations further. A number of physiotherapist or parent-led physical therapy interventions shortly after birth have been shown to improve bone mass and size in pediatric groups prone to low neonatal bone mass [44-48] and may be applicable in children born with breech presentation. These early interventions may be particularly effective as they coincide with a rapid period of skeletal growth [2]. There is some evidence that advantages in bone size gained through skeletal loading in childhood persist throughout life [49], whereas at the end of adolescence the ability to increase bone size is markedly reduced or even absent [50]. It may also be informative to categorize deliveries according to type of breech presentation in future work. Frank breech presentation (hips flexed, knees extended) is the most common breech position [51], but complete (hips flexed, knees flexed) or incomplete/footling breech (legs extended) positions also occur, possibly with different musculoskeletal consequences. For example, there is some limited evidence that knee position may affect joint development [18] but this remains unexplored in a large cohort of children with breech presentation.

\section{Strengths and weaknesses}

This study examined a large cohort with prospective information on a number of potential confounding factors. However, as an observational study causality cannot be attributed. Only a limited portion of the original cohort had complete exposure, outcome and covariable data and differences in cohort characteristics between included and excluded participants may have introduced selection bias. Our exploration of potential mediators including physical activity and diet was limited by availability of information collected. We had limited statistical power, with few participants having a breech presentation. Hence, several of our association estimates are relatively imprecise, with wide confidence intervals. Furthermore, as this is the first study of these associations replication in larger studies is required. Like other previous studies of breech presentation and bone outcomes, fetal position was not recorded throughout pregnancy. Around $45 \%$ of fetuses are in a breech presentation at 25 weeks [52], followed by a roughly linear decline through to $4-5 \%$ at term. Although this study only measured breech presentation at a single point at delivery, this is likely to have detected those in breech for the longest time as the incidence of spontaneous cephalic-breech position decreases with gestation [53]. Therefore, this would not have necessarily prevented us from finding an association with hip BMD assuming earlier onset and longer duration of breech presentation has greater effects. On the other hand, differences could have been attenuated by misclassification bias whereby those with significant duration of breech presentation who correct shortly before birth are classified as cephalic. We are assuming that breech presentation influences bone outcomes because it limits fetal movements and future studies with direct measurements of fetal movement, together with long-term follow-up, are required to determine this. Unlike DXA-based measures, associations between PQCT variables and long-term fracture risk are not well-established. Therefore we were 
unable to extrapolate observed group differences in bone outcomes into differences in predicted fracture risk.

\section{Conclusions}

Breech presentation was associated with lower adolescent bone mass, area, density and strength in the tibia, and with lower hip area in females. These associations were not attenuated by adjustment for potential confounders or mediators. Breech presentation may therefore represent a modifiable risk factor for low bone mass and size, although given the relatively small sample size these findings require replication. Larger, more detailed studies of fetal presentation and movement throughout pregnancy could identify possible key periods and causal factors, with the aim of informing strategies for reducing long term health consequences of breech delivery.

\section{Acknowledgements}

We are extremely grateful to all the families who took part in this study, the midwives for their help in recruiting them, and the whole ALSPAC team, which includes interviewers, computer and laboratory technicians, clerical workers, research scientists, volunteers, managers, receptionists and nurses.

\section{Funding}

The UK Medical Research Council and Wellcome (Grant ref: 102215/2/13/2) and the University of Bristol provide core support for ALSPAC. DXA and PQCT scans were funded by Wellcome grant WT084632. A comprehensive list of ALSPAC grants funding is available on the ALSPAC website (http://www.bristol.ac.uk/alspac/external/documents/grant-acknowledgements.pdf). DAL works in a Unit that receives support from the UK Medical Research Council (MC_UU_00011/6) and the University of Bristol and in the Bristol National Institute for Health Research funded Biomedical Research Centre. DAL is a National Institute for Health Research Senior Investigator (NF-SI-061110196). No funders had any role in data collection, analyses or interpretation of findings. This publication is the work of the authors and the opinions expressed here do not necessarily reflect those of the funders. Jon Tobias and Alex Ireland will serve as guarantors for the contents of this paper. 


\section{References}

1. Hernandez CJ, Beaupré GS, Carter DR (2003) A theoretical analysis of the relative influences of peak BMD, age-related bone loss and menopause on the development of osteoporosis.

Osteoporos Int 14:843-847

2. Ruff C (2003) Growth in bone strength, body size, and muscle size in a juvenile longitudinal sample Bone 33:317-329

3. Foley S, Quinn S, Jones G (2009) Tracking of bone mass from childhood to adolescence and factors that predict deviation from tracking. Bone 44:752-757

4. Harvey NC, Javaid MK, Arden NK, et al. (2010) Maternal predictors of neonatal bone size and geometry: the Southampton Women's Survey. J Dev Orig Health Dis 1:35-41

5. Steer CD, Sayers A, Kemp J, Fraser WD, Tobias JH (2014) Birth weight is positively related to bone size in adolescents but inversely related to cortical bone mineral density: findings from a large prospective cohort study. Bone 65:77-82

6. Dennison EM, Syddall HE, Sayer AA, Gilbody HJ, Cooper C (2005) Birth weight and weight at 1 year are independent determinants of bone mass in the seventh decade: the Hertfordshire cohort study. Pediatr Res 57:582-586

7. Cooper C, Eriksson JG, Forsén T, Osmond C, Tuomilehto J, Barker DJ (2001) Maternal height, childhood growth and risk of hip fracture in later life: a longitudinal study. Osteoporos Int 12:623629

8. Ireland A, Rittweger J, Degens H (2013) The Influence of Muscular Action on Bone Strength Via Exercise. Clinical Reviews in Bone and Mineral Metabolism 12:93-102

9. Giorgi M, Carriero A, Shefelbine SJ, Nowlan NC (2015) Effects of normal and abnormal loading conditions on morphogenesis of the prenatal hip joint: application to hip dysplasia. J Biomech 48:3390-3397

10. Nowlan NC, Sharpe J, Roddy KA, Prendergast PJ, Murphy P (2010) Mechanobiology of embryonic skeletal development: Insights from animal models. Birth Defects Res C Embryo Today 90:203-213

11. Sival DA, Prechtl HF, Sonder GH, Touwen BC (1993) The effect of intra-uterine breech position on postnatal motor functions of the lower limbs. Early Hum Dev 32:161-176

12. Fong BF, Savelsbergh GJ, de Vries JI (2009) Fetal leg posture in uncomplicated breech and cephalic pregnancies. Eur J Pediatr 168:443-447

13. Luterkort M, Marsál K (1985) Fetal motor activity in breech presentation. Early Hum Dev 10:193-200

14. Verbruggen SW, Kainz B, Shelmerdine SC, Arthurs OJ, Hajnal JV, Rutherford MA, Phillips ATM, Nowlan NC (2018) Altered biomechanical stimulation of the developing hip joint in presence of hip dysplasia risk factors. J Biomech

15. Verbruggen SW, Kainz B, Shelmerdine SC, Hajnal JV, Rutherford MA, Arthurs OJ, Phillips AT, Nowlan NC (2018) Stresses and strains on the human fetal skeleton during development. Journal of The Royal Society Interface

16. Chan A, McCaul KA, Cundy PJ, Haan EA, Byron-Scott R (1997) Perinatal risk factors for developmental dysplasia of the hip. Arch Dis Child Fetal Neonatal Ed 76:F94-100

17. Hinderaker T, Uden A, Reikerås O (1994) Direct ultrasonographic measurement of femoral anteversion in newborns. Skeletal Radiol 23:133-135

18. Øye CR, Foss OA, Holen KJ (2016) Breech presentation is a risk factor for dysplasia of the femoral trochlea. Acta Orthop 87:17-21

19. Ireland A, Crozier S, Heazell A, Ward K, Godfrey K, Inskip H, Cooper C, Harvey N (2018) Breech presentation is associated with lower bone mass and area: findings from the Southampton Women's Survey. Osteoporosis International 
20. Sekulić S, Zarkov M, Slankamenac P, Bozić K, Vejnović T, Novakov-Mikić A (2009) Decreased expression of the righting reflex and locomotor movements in breech-presenting newborns in the first days of life. Early Hum Dev 85:263-266

21. Bartlett DJ, Okun NB, Byrne PJ, Watt JM, Piper MC (2000) Early motor development of breech- and cephalic-presenting infants. Obstet Gynecol 95:425-432

22. Fong BF, Savelsbergh GJ, Leijsen MR, de Vries JI (2009) The influence of prenatal breech presentation on neonatal leg posture. Early Hum Dev 85:201-206

23. Herlitz $G$ (1959) Limitation of movement of the hip joints in new-born infants following breech presentation. Acta Paediatr Suppl 48:123-125

24. Kean LH, Suwanrath C, Gargari SS, Sahota DS, James DK (1999) A comparison of fetal behaviour in breech and cephalic presentations at term. Br J Obstet Gynaecol 106:1209-1213 25. Fong B, Ledebt A, Zwart R, De Vries JI, Savelsbergh GJ (2008) Is there an effect of prenatal breech position on locomotion at 2.5 years? Early Hum Dev 84:211-216

26. Miller E, Kouam L (1981) Zur Haufigkeit von Beckenendlagen im Verlauf

Der Schwangerschaft und zum Zeitpunkt der Geburt Zentralbl Gynakol 103:105-109

27. Cammu H, Dony N, Martens G, Colman R (2014) Common determinants of breech presentation at birth in singletons: a population-based study. Eur J Obstet Gynecol Reprod Biol 177:106-109

28. Boyd A, Golding J, Macleod J, Lawlor DA, Fraser A, Henderson J, Molloy L, Ness A, Ring S, Davey Smith G (2013) Cohort Profile: the 'children of the 90s'--the index offspring of the Avon Longitudinal Study of Parents and Children. Int J Epidemiol 42:111-127

29. Fraser A, Macdonald-Wallis C, Tilling K, et al. (2013) Cohort Profile: the Avon Longitudinal Study of Parents and Children: ALSPAC mothers cohort. Int J Epidemiol 42:97-110

30. Ward KA, Adams JE, Hangartner TN (2005) Recommendations for thresholds for cortical bone geometry and density measurement by peripheral quantitative computed tomography. Calcif Tissue Int 77:275-280

31. Frankenburg WK, Dodds JB (1967) The Denver developmental screening test. J Pediatr 71:181-191

32. Tshorny M, Mimouni FB, Littner Y, Alper A, Mandel D (2007) Decreased neonatal tibial bone ultrasound velocity in term infants born after breech presentation. J Perinatol 27:693-696

33. Ireland A, Sayers A, Deere KC, Emond A, Tobias JH (2016) Motor Competence in Early Childhood Is Positively Associated With Bone Strength in Late Adolescence. J Bone Miner Res 31:1089-1098

34. Bartlett D, Okun N (1994) Breech presentation: a random event or an explainable phenomenon? Dev Med Child Neurol 36:833-838

35. Andersen GL, Irgens LM, Skranes J, Salvesen KA, Meberg A, Vik T (2009) Is breech presentation a risk factor for cerebral palsy? A Norwegian birth cohort study. Dev Med Child Neurol 51:860-865

36. Luterkort M, Persson PH, Weldner BM (1984) Maternal and fetal factors in breech presentation. Obstet Gynecol 64:55-59

37. Verbruggen SW, Kainz B, Shelmerdine SC, Hajnal JV, Rutherford MA, Arthurs OJ, Phillips AT, Nowlan NC (2018) Stresses and strains on the human fetal skeleton during development. Journal of The Royal Society Interface 15:20170593

38. Andersson JE, Vogel I, Uldbjerg N (2002) Serum 17 beta-estradiol in newborn and neonatal hip instability. J Pediatr Orthop 22:88-91

39. Vanderschueren D, Venken K, Ophoff J, Bouillon R, Boonen S (2006) Clinical Review: Sex steroids and the periosteum--reconsidering the roles of androgens and estrogens in periosteal expansion. J Clin Endocrinol Metab 91:378-382

40. Ireland A, Rittweger J, Schönau E, Lamberg-Allardt C, Viljakainen H (2014) Time Since Onset of Walking Predicts Tibial Bone Strength in Early Childhood. Bone 68:76-84 
41. Cooper C, Walker-Bone K, Arden N, Dennison E (2000) Novel insights into the pathogenesis of osteoporosis: the role of intrauterine programming. Rheumatology (Oxford) 39:1312-1315

42. Hofmeyr GJ, Kulier R, West HM (2015) External cephalic version for breech presentation at term. Cochrane Database Syst Rev CD000083

43. Lambeek AF, De Hundt M, Vlemmix F, Akerboom BM, Bais JM, Papatsonis DN, Mol BW, Kok $M$ (2013) Risk of developmental dysplasia of the hip in breech presentation: the effect of successful external cephalic version. BJOG 120:607-612

44. Litmanovitz I, Dolfin T, Arnon S, Regev RH, Nemet D, Eliakim A (2007) Assisted exercise and bone strength in preterm infants. Calcif Tissue Int 80:39-43

45. Litmanovitz I, Dolfin T, Friedland O, Arnon S, Regev R, Shainkin-Kestenbaum R, Lis M, Eliakim A (2003) Early physical activity intervention prevents decrease of bone strength in very low birth weight infants. Pediatrics 112:15-19

46. Moyer-Mileur LJ, Ball SD, Brunstetter VL, Chan GM (2008) Maternal-administered physical activity enhances bone mineral acquisition in premature very low birth weight infants. J Perinatol 28:432-437

47. Moyer-Mileur LJ, Brunstetter V, McNaught TP, Gill G, Chan GM (2000) Daily physical activity program increases bone mineralization and growth in preterm very low birth weight infants.

Pediatrics 106:1088-1092

48. Vignochi CM, Miura E, Canani LH (2008) Effects of motor physical therapy on bone mineralization in premature infants: a randomized controlled study. J Perinatol 28:624-631

49. Warden SJ, Mantila Roosa SM, Kersh ME, Hurd AL, Fleisig GS, Pandy MG, Fuchs RK (2014) Physical activity when young provides lifelong benefits to cortical bone size and strength in men. Proc Natl Acad Sci U S A 111:5337-5342

50. Ireland A, Maden-Wilkinson T, Ganse B, Degens H, Rittweger J (2014) Effects of age and starting age upon side asymmetry in the arms of veteran tennis players: a cross-sectional study. Osteoporos Int 25:1389-1400

51. Hannah ME, Hannah WJ, Hewson SA, Hodnett ED, Saigal S, Willan AR (2000) Planned caesarean section versus planned vaginal birth for breech presentation at term: a randomised multicentre trial. Term Breech Trial Collaborative Group. Lancet 356:1375-1383

52. Suzuki S, Yamamuro T (1985) Fetal movement and fetal presentation. Early Hum Dev 11:255-

263

53. Witkop CT, Zhang J, Sun W, Troendle J (2008) Natural history of fetal position during pregnancy and risk of nonvertex delivery. Obstet Gynecol 111:875-880 


\begin{tabular}{|c|c|c|c|c|c|c|}
\hline \multirow{3}{*}{\multicolumn{2}{|c|}{ Participant variable }} & \multicolumn{4}{|c|}{ Presentation at Onset of Labour } & \multirow{3}{*}{$\begin{array}{l}P \text {-value for } \\
\text { group } \\
\text { comparison }\end{array}$} \\
\hline & & \multicolumn{2}{|c|}{ Cephalic } & \multicolumn{2}{|c|}{ Breech } & \\
\hline & & $n$ & $\%$ & $n$ & $\%$ & \\
\hline \multicolumn{2}{|l|}{$\mathrm{n}$} & 1869 & $94.8 \%$ & 102 & $5.2 \%$ & - \\
\hline \multicolumn{2}{|l|}{ Females } & 1000 & $53.5 \%$ & 62 & $60.8 \%$ & 0.155 \\
\hline \multirow{3}{*}{ Caesarean Delivery } & No & 1646 & $88.1 \%$ & 24 & $23.5 \%$ & \multirow[t]{3}{*}{$<0.001$} \\
\hline & Elective & 67 & $3.6 \%$ & 34 & $33.3 \%$ & \\
\hline & Emergency & 156 & $8.3 \%$ & 44 & $43.1 \%$ & \\
\hline \multicolumn{2}{|c|}{ Maternal Smoking During Pregnancy } & 291 & $15.6 \%$ & 22 & $21.6 \%$ & 0.125 \\
\hline \multicolumn{2}{|c|}{ Singleton birth } & 1812 & $97.0 \%$ & 95 & $93.1 \%$ & 0.445 \\
\hline \multirow{4}{*}{ Parity } & 0 & 1020 & $54.6 \%$ & 65 & $63.7 \%$ & \multirow[t]{4}{*}{0.402} \\
\hline & 1 & 568 & $30.4 \%$ & 28 & $27.5 \%$ & \\
\hline & 2 & 214 & $11.4 \%$ & 6 & $5.9 \%$ & \\
\hline & $\geq 3$ & 67 & $3.6 \%$ & 3 & $2.9 \%$ & \\
\hline \multirow{5}{*}{ Maternal Education } & Up to CSE & 164 & $8.8 \%$ & 8 & $7.8 \%$ & \multirow[t]{5}{*}{0.829} \\
\hline & Vocational & 141 & $7.5 \%$ & 9 & $8.8 \%$ & \\
\hline & O Level & 767 & $41.0 \%$ & 45 & $44.1 \%$ & \\
\hline & A Level & 334 & $17.9 \%$ & 14 & $13.7 \%$ & \\
\hline & Degree & 463 & $24.8 \%$ & 26 & $25.5 \%$ & \\
\hline & Mean & SD & Mean & SD & \\
\hline \multicolumn{2}{|l|}{ Birthweight (g) } & 3396 & 554 & 3036 & 745 & $<0.001$ \\
\hline \multicolumn{2}{|l|}{ Gestation age (weeks) } & 39.4 & 1.9 & 38.1 & 2.9 & $<0.001$ \\
\hline \multicolumn{2}{|l|}{ Maternal Age (years) } & 29.5 & 4.6 & 30.3 & 4.3 & 0.095 \\
\hline \multicolumn{2}{|c|}{ Gross Motor Score at 18 months } & 19.4 & 2.8 & 18.4 & 3.4 & 0.252 \\
\hline \multicolumn{2}{|c|}{ Age at $17 y$ check (years) } & 17.8 & 0.4 & 17.8 & 0.4 & 0.37 \\
\hline \multicolumn{2}{|l|}{ Height at $17 y(\mathrm{~cm})$} & 172 & 9 & 171 & 9 & 0.288 \\
\hline \multicolumn{2}{|l|}{ Body mass at $17 y(\mathrm{~kg})$} & 65.4 & 11.6 & 65.1 & 11.2 & 0.75 \\
\hline
\end{tabular}

Table 1. Cohort characteristics separated by presentation 


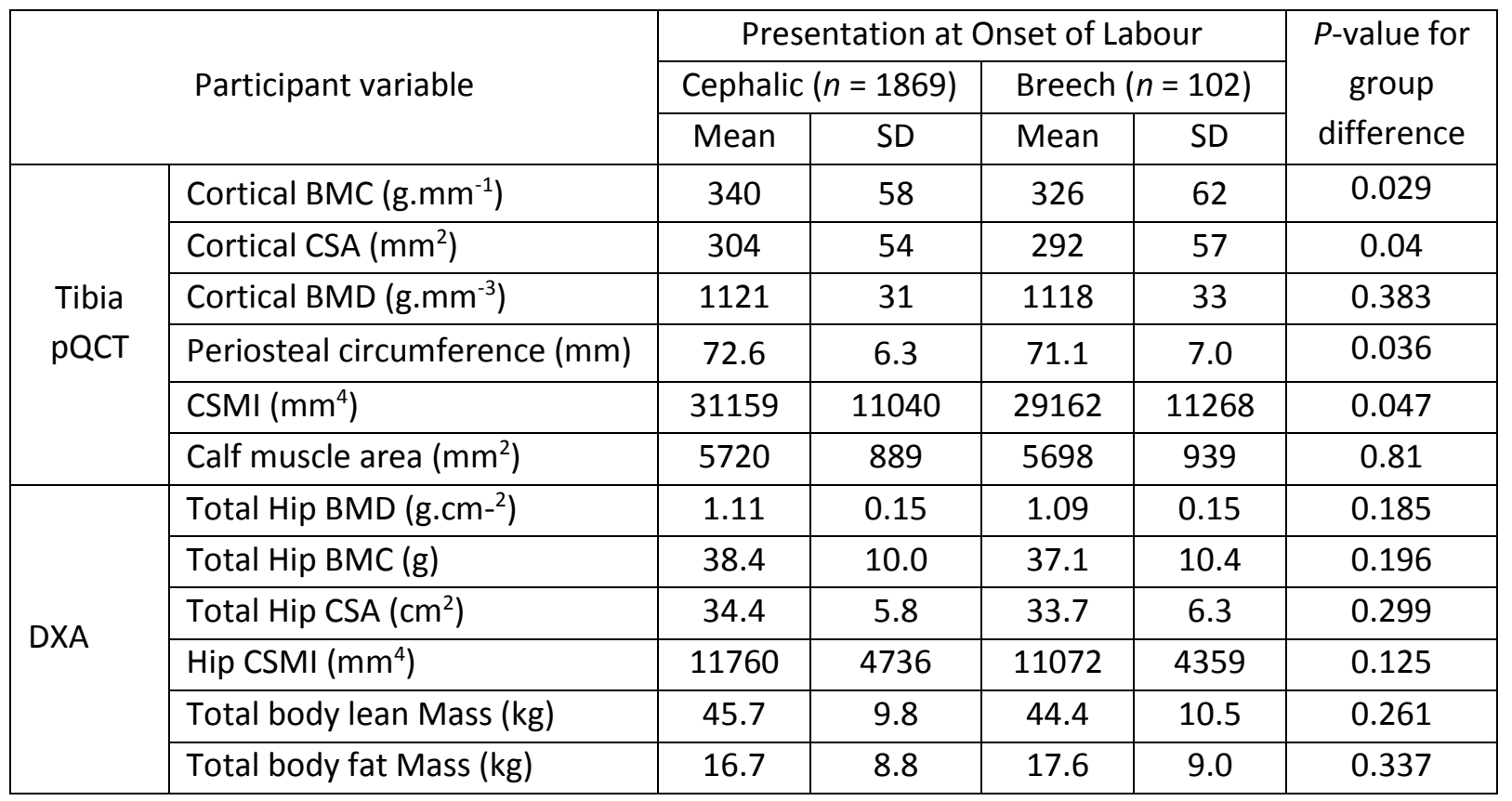

Table 2. Bone outcomes and body composition separated by presentation. BMC - bone mineral content, CSA - cross-sectional area, BMD - bone mineral density, CSMI - cross-sectional moment of inertia 


\begin{tabular}{|c|c|c|c|c|c|c|c|c|c|c|c|c|c|c|c|c|}
\hline \multirow{2}{*}{\multicolumn{2}{|c|}{ Variable }} & \multirow{3}{*}{$\begin{array}{c}\text { Group } \\
\text { Combined }\end{array}$} & \multicolumn{4}{|c|}{ Model 1} & \multicolumn{4}{|c|}{ Model 2} & \multirow{5}{*}{$\begin{array}{l}\text { Int } P \\
0.992\end{array}$} & \multicolumn{4}{|c|}{ Model 3} & \multirow{3}{*}{ Int $P$} \\
\hline & & & \multirow{2}{*}{$\begin{array}{c}\text { Beta } \\
-0.091\end{array}$} & \multicolumn{2}{|c|}{$95 \% \mathrm{Cl}$} & \multirow{2}{*}{$\begin{array}{c}P \\
0.333\end{array}$} & \multirow{2}{*}{$\begin{array}{c}\text { Beta } \\
-0.074\end{array}$} & \multicolumn{2}{|c|}{$95 \% \mathrm{Cl}$} & \multirow{2}{*}{$\begin{array}{c}P \\
0.426\end{array}$} & & \multirow{2}{*}{$\begin{array}{c}\text { Beta } \\
-0.086\end{array}$} & \multicolumn{2}{|c|}{$95 \% \mathrm{Cl}$} & \multirow{2}{*}{$\begin{array}{c}P \\
0.39\end{array}$} & \\
\hline \multirow{12}{*}{ DXA } & \multirow{3}{*}{$\begin{array}{l}\text { Total } \\
\text { Hip } \\
\text { BMD }\end{array}$} & & & -0.277 & 0.094 & & & -0.258 & 0.109 & & & & -0.281 & 0.11 & & \\
\hline & & Male & & & & & -0.106 & -0.424 & 0.212 & 0.515 & & -0.127 & -0.461 & 0.206 & 0.454 & \multirow[t]{2}{*}{0.83} \\
\hline & & Female & & & & & -0.084 & -0.339 & 0.171 & 0.519 & & -0.138 & -0.414 & 0.137 & 0.325 & \\
\hline & \multirow{3}{*}{$\begin{array}{l}\text { Total } \\
\text { Hip } \\
\text { BMC }\end{array}$} & Combined & -0.059 & -0.198 & 0.079 & 0.411 & -0.032 & -0.159 & 0.096 & 0.628 & \multirow{3}{*}{0.185} & -0.049 & -0.184 & 0.086 & 0.475 & \multirow{3}{*}{0.28} \\
\hline & & Male & & & & & 0.048 & -0.24 & 0.336 & 0.745 & & -0.001 & -0.301 & 0.298 & 0.993 & \\
\hline & & Female & & & & & -0.187 & -0.419 & 0.046 & 0.116 & & -0.217 & -0.465 & 0.031 & 0.089 & \\
\hline & \multirow{3}{*}{$\begin{array}{l}\text { Total } \\
\text { Hip } \\
\text { CSA }\end{array}$} & Combined & -0.026 & -0.142 & 0.09 & 0.661 & 0.004 & -0.097 & 0.105 & 0.94 & \multirow{3}{*}{0.006} & -0.019 & -0.128 & 0.09 & 0.735 & \multirow{3}{*}{0.01} \\
\hline & & Male & & & & & 0.211 & -0.043 & 0.466 & 0.104 & & 0.143 & -0.128 & 0.414 & 0.302 & \\
\hline & & Female & & & & & -0.215 & -0.427 & -0.004 & 0.046 & & -0.196 & -0.428 & 0.036 & 0.098 & \\
\hline & \multirow{3}{*}{$\begin{array}{l}\text { Hip } \\
\text { CSMI }\end{array}$} & Combined & -0.066 & -0.202 & 0.07 & 0.343 & -0.044 & -0.171 & 0.082 & 0.491 & \multirow{3}{*}{0.359} & -0.04 & -0.174 & 0.094 & 0.559 & \multirow{3}{*}{0.29} \\
\hline & & Male & & & & & -0.17 & -0.45 & 0.111 & 0.236 & & -0.101 & -0.395 & 0.192 & 0.499 & \\
\hline & & Female & & & & & -0.003 & -0.237 & 0.23 & 0.977 & & -0.125 & -0.372 & 0.121 & 0.319 & \\
\hline
\end{tabular}

Table 3. Associations between breech presentation and DXA-derived bone measures. Results are presented for both sexes combined, and for Models 2 and 3 (where adjustment for sex was included) sex-stratified results and sex*presentation interactions (Int $P$ ) are also reported. Standardized regression coefficients (Beta) represent the difference in mean bone outcomes relative to cephalic presentation in SD, 95\% Cls and $P$ values. Adjustments: Model 1 : height; Model 2: Model $1+$ sex, age at outcome, maternal social class, maternal smoking, delivery method, parity, maternal age and singleton/multiple births; Model 3: Model $2+$ total body fat mass, calf muscle CSA, gestation length, birthweight, delivery method and Gross Motor Score. 


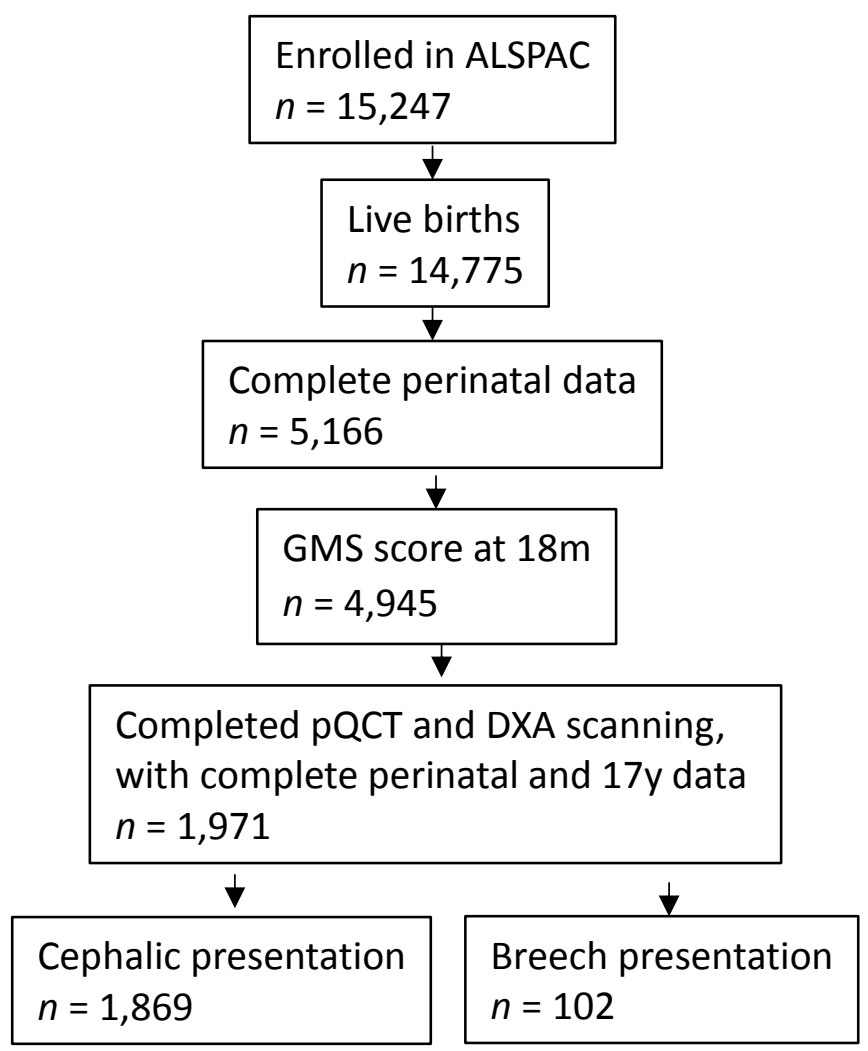

Figure 1. Flow diagram showing participant $\mathrm{n}$ at each stage of data preparation. 

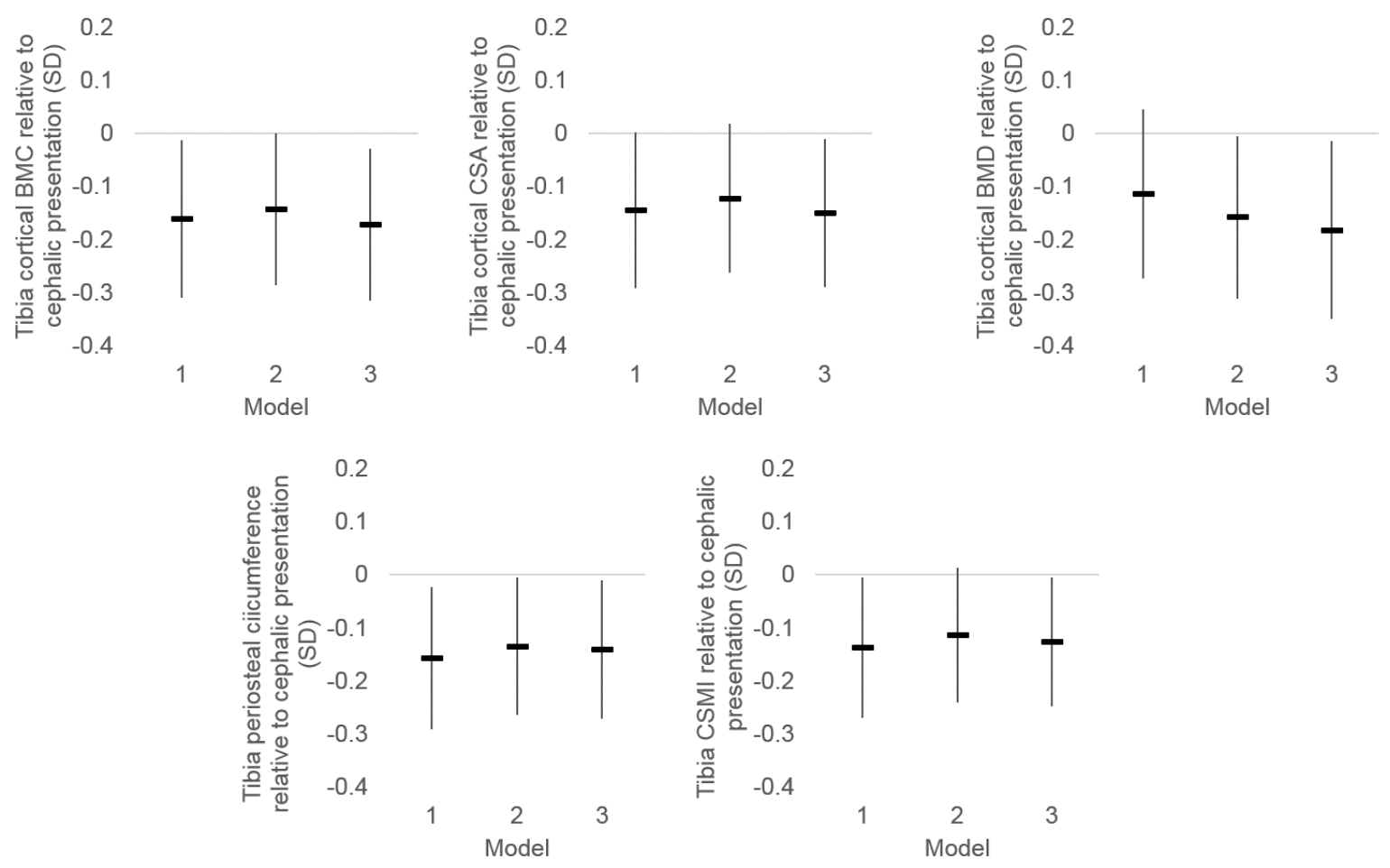

Figure 2. Associations between breech presentation and pQCT-derived bone measures in 1971 participants (1062 females). Data points are standardized regression coefficients representing the difference in mean bone outcomes relative to cephalic presentation in SD plus 95\% Cls. Adjustments: Model 1: height; Model 2: Model $1+$ sex, age at outcome, maternal social class, maternal smoking, parity, maternal age and singleton/multiple births; Model 3: Model $2+$ total body fat mass, calf muscle CSA, gestation length, birthweight, delivery method and Gross Motor Score. 


\begin{tabular}{|c|c|c|c|c|c|c|}
\hline \multirow{3}{*}{\multicolumn{2}{|c|}{ Participant variable }} & \multicolumn{4}{|c|}{ Data } & \multirow{3}{*}{$\begin{array}{l}P \text {-value for } \\
\text { group } \\
\text { comparison }\end{array}$} \\
\hline & & \multicolumn{2}{|c|}{ Complete } & \multicolumn{2}{|c|}{ Incomplete } & \\
\hline & & $n$ & $\%$ & $n$ & $\%$ & \\
\hline \multicolumn{2}{|l|}{$\mathrm{n}$} & 1971 & $38.2 \%$ & 3195 & $61.8 \%$ & - \\
\hline \multicolumn{2}{|c|}{ Females } & 1062 & $53.9 \%$ & 1424 & $44.6 \%$ & $<0.001$ \\
\hline \multicolumn{2}{|c|}{ Breech Presentation } & 102 & $5.2 \%$ & 174 & $5.4 \%$ & 0.721 \\
\hline \multirow{3}{*}{ Caesarean Delivery } & No & 1670 & $84.7 \%$ & 2651 & $83.0 \%$ & \multirow[t]{3}{*}{0.051} \\
\hline & Elective & 101 & $5.1 \%$ & 217 & $6.8 \%$ & \\
\hline & Emergency & 200 & $10.1 \%$ & 221 & $6.9 \%$ & \\
\hline \multicolumn{2}{|c|}{ Maternal Smoking During Pregnancy } & 313 & $15.9 \%$ & 780 & $24.4 \%$ & $<0.001$ \\
\hline \multicolumn{2}{|c|}{ Singleton birth (Yes/No) } & 1907 & $96.8 \%$ & 3071 & $96.1 \%$ & 0.941 \\
\hline \multirow{4}{*}{ Parity } & 0 & 1085 & $55.0 \%$ & 1520 & $47.6 \%$ & \multirow[t]{4}{*}{0.009} \\
\hline & 1 & 596 & $30.2 \%$ & 984 & $30.8 \%$ & \\
\hline & 2 & 220 & $11.2 \%$ & 382 & $12.0 \%$ & \\
\hline & $\geq 3$ & 70 & $3.6 \%$ & 148 & $4.6 \%$ & \\
\hline \multirow{5}{*}{ Maternal Education } & Up to CSE & 172 & $8.7 \%$ & 551 & $17.2 \%$ & \multirow[t]{5}{*}{$<0.001$} \\
\hline & Vocational & 150 & $7.6 \%$ & 291 & $9.1 \%$ & \\
\hline & O Level & 812 & $41.2 \%$ & 1415 & $44.3 \%$ & \\
\hline & A Level & 348 & $17.7 \%$ & 463 & $14.5 \%$ & \\
\hline & Degree & 489 & $24.8 \%$ & 475 & $14.9 \%$ & \\
\hline & Mean & SD & Mean & SD & \\
\hline \multicolumn{2}{|c|}{ Birthweight (g) } & 3377 & 571 & 3380 & 583 & 0.865 \\
\hline \multicolumn{2}{|c|}{ Gestation age (weeks) } & 39.3 & 2.0 & 39.3 & 2.1 & 0.622 \\
\hline \multicolumn{2}{|c|}{ Maternal Age (years) } & 29.6 & 4.6 & 28.1 & 4.8 & $<0.001$ \\
\hline
\end{tabular}

Supplementary Table 1. Characteristics of participants with complete data included in current study and those with incomplete data excluded from study 


\begin{tabular}{|c|c|c|c|c|c|c|c|c|c|c|c|c|c|c|c|c|}
\hline & \multirow{2}{*}{ Variable } & \multirow{3}{*}{$\begin{array}{c}\text { Sex } \\
\text { Combined }\end{array}$} & \multicolumn{4}{|c|}{ Model 1} & \multicolumn{4}{|c|}{ Model 2} & \multirow{2}{*}{$\operatorname{lnt} P$} & \multicolumn{4}{|c|}{ Model 3} & \multirow{2}{*}{$\begin{array}{c}\text { Int } \\
P\end{array}$} \\
\hline & & & \multirow{2}{*}{$\begin{array}{c}\text { Beta } \\
-0.162 \\
\end{array}$} & \multicolumn{2}{|c|}{$95 \% \mathrm{Cl}$} & \multirow{2}{*}{$\begin{array}{c}P \\
0.033\end{array}$} & \multirow{2}{*}{$\begin{array}{c}\text { Beta } \\
-0.144\end{array}$} & \multicolumn{2}{|c|}{$95 \% \mathrm{Cl}$} & \multirow{2}{*}{\begin{tabular}{|c|}
$P$ \\
0.050 \\
\end{tabular}} & & \multirow{2}{*}{$\begin{array}{c}\text { Beta } \\
-0.175\end{array}$} & \multicolumn{2}{|c|}{$95 \% \mathrm{Cl}$} & \multirow{2}{*}{$\begin{array}{c}P \\
0.017 \\
\end{array}$} & \\
\hline \multirow{15}{*}{$\begin{array}{l}\text { Tibia } \\
\text { pQCT }\end{array}$} & & & & -0.310 & -0.013 & & & -0.288 & 0.000 & & \multirow{3}{*}{0.542} & & -0.318 & -0.031 & & \multirow{3}{*}{0.88} \\
\hline & \multirow[t]{2}{*}{ Cortical BMC } & Male & & & & & -0.114 & -0.402 & 0.174 & 0.438 & & -0.171 & -0.452 & 0.111 & 0.236 & \\
\hline & & Female & & & & & -0.250 & -0.472 & -0.027 & 0.028 & & -0.315 & -0.541 & -0.090 & 0.006 & \\
\hline & \multirow{3}{*}{ Cortical CSA } & Combined & -0.146 & -0.292 & 0.001 & 0.052 & -0.123 & -0.263 & 0.018 & 0.087 & \multirow{3}{*}{0.578} & -0.150 & -0.290 & -0.011 & 0.035 & \multirow{3}{*}{0.95} \\
\hline & & Male & & & & & -0.101 & -0.393 & 0.192 & 0.501 & & -0.171 & -0.457 & 0.116 & 0.243 & \\
\hline & & Female & & & & & -0.232 & -0.462 & -0.003 & 0.047 & & -0.284 & -0.516 & -0.051 & 0.017 & \\
\hline & \multirow{3}{*}{ Cortical BMD } & Combined & -0.115 & -0.275 & 0.045 & 0.158 & -0.159 & -0.312 & -0.006 & 0.014 & \multirow{3}{*}{0.398} & -0.183 & -0.351 & -0.015 & 0.033 & \multirow{3}{*}{0.38} \\
\hline & & Male & & & & & -0.067 & -0.358 & 0.224 & 0.652 & & -0.005 & -0.319 & 0.310 & 0.977 & \\
\hline & & Female & & & & & -0.212 & -0.410 & -0.014 & 0.036 & & -0.336 & -0.557 & -0.115 & 0.003 & \\
\hline & \multirow{3}{*}{$\begin{array}{c}\text { Periosteal } \\
\text { Circumference }\end{array}$} & Combined & -0.158 & -0.292 & -0.023 & 0.021 & -0.135 & -0.265 & -0.006 & 0.041 & \multirow{3}{*}{0.295} & -0.142 & -0.273 & -0.011 & 0.034 & \multirow{3}{*}{0.56} \\
\hline & & Male & & & & & -0.080 & -0.360 & 0.199 & 0.573 & & -0.117 & -0.393 & 0.158 & 0.404 & \\
\hline & & Female & & & & & -0.271 & -0.491 & -0.050 & 0.017 & & -0.275 & -0.504 & -0.046 & 0.019 & \\
\hline & \multirow{3}{*}{ CSMI } & Combined & -0.138 & -0.270 & -0.006 & 0.040 & -0.114 & -0.241 & 0.013 & 0.075 & \multirow{3}{*}{0.389} & -0.128 & -0.249 & -0.008 & 0.037 & \multirow{3}{*}{0.65} \\
\hline & & Male & & & & & -0.077 & -0.356 & 0.202 & 0.588 & & -0.094 & -0.359 & 0.170 & 0.485 & \\
\hline & & Female & & & & & -0.212 & -0.431 & 0.008 & 0.059 & & -0.255 & -0.470 & -0.040 & 0.020 & \\
\hline
\end{tabular}

Supplementary Table 2. Associations and sex interactions (Int P) between breech presentation and tibia pQCT bone measures as combined data and stratified by sex for Models 1, 2 and 3. Standardized regression coefficients (Beta) represent the mean difference in bone outcomes relative to cephalic presentation in SD, 95\% Cls and $P$ values. Adjustments: Model 1: Height; Model 2: Model $1+$ sex, age at outcome, maternal social class, maternal smoking, parity, maternal age and singleton/multiple births; Model 3: Model 2 + gestation length, birthweight, delivery method, Gross Motor Score, fat mass and lean mass (or calf muscle CSA for PQCT outcomes). 

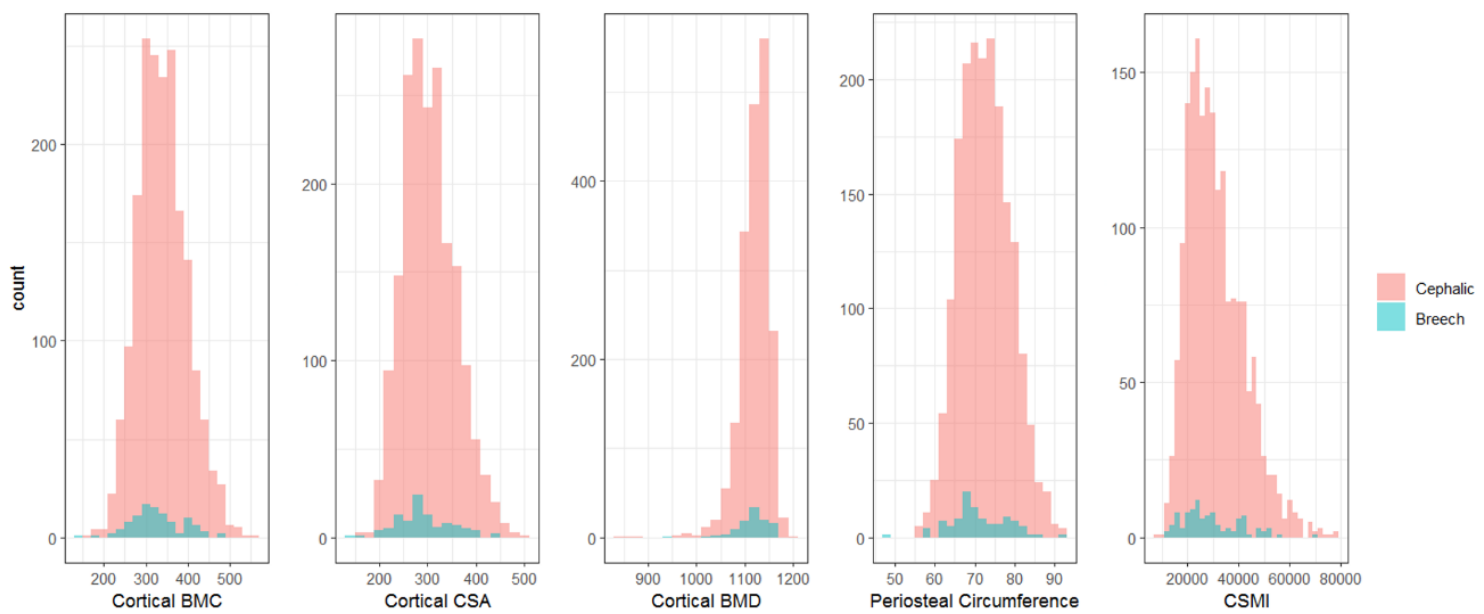

Supplementary Figure 1. Histograms of tibia bone outcomes separated by presentation, for which associations with breech presentation were observed. 


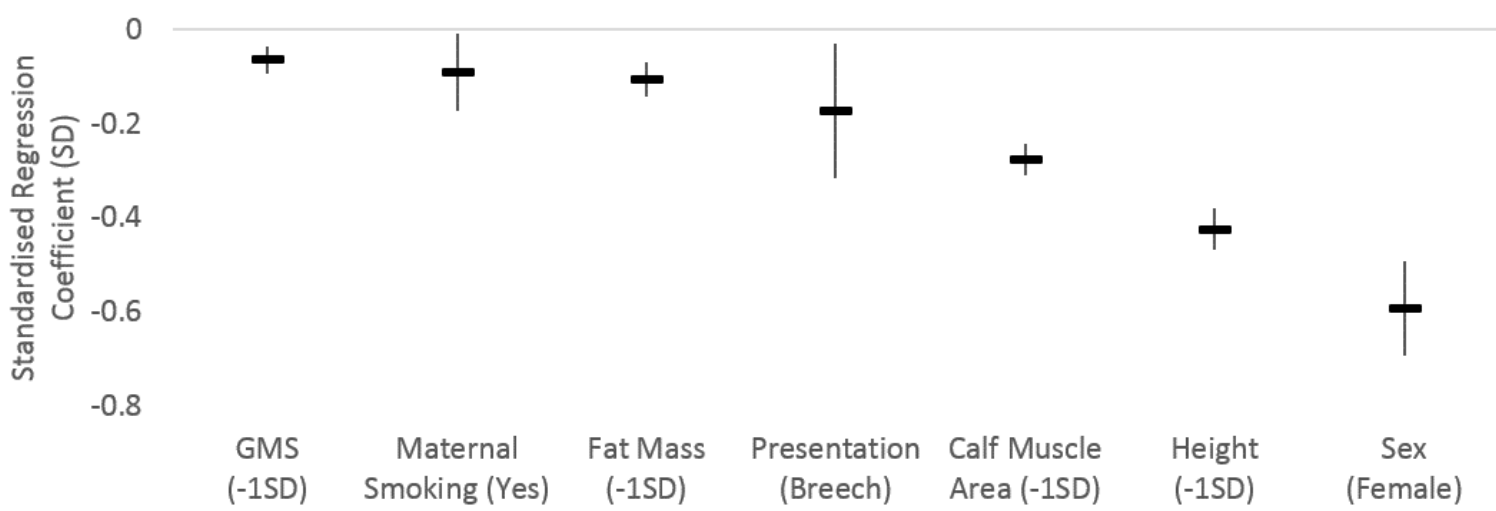

Supplementary Figure 2. Associations of breech presentation and covariables with tibia cortical BMC identified within Model 3 in this study, ordered by decreasing regression coefficient; error bars indicate $95 \% \mathrm{Cl}$. GMS - Gross Motor Score. 